\title{
Evaluation of a Primary Care-Based Post-Discharge Phone Call Program: Keeping the Primary Care Practice at the Center of Post-hospitalization Care Transition
}

\author{
Ning Tang, $M D^{7}$, Jeffrey Fujimoto, $B S^{1,2}$, and Leah Karliner, $M D^{1,3}$ \\ 'Division of General Internal Medicine, Department of Medicine, University of California San Francisco, San Francisco, CA, USA; ${ }^{2}$ David \\ Geffen School of Medicine, University of California Los Angeles, Los Angeles, CA, USA; ${ }^{3}$ Medical Effectiveness Research Center for Diverse \\ Populations, University of California San Francisco, San Francisco, CA, USA.
}

BACKGROUND: The post-hospitalization period is a precarious time for patients. Post-discharge nurse telephone call programs aiming to prevent unnecessary readmissions have had mixed results.

OBJECTIVE: Describe a primary-care based program to identify and address problems arising after hospital discharge.

DESIGN: A quality improvement program embedding registered nurses in a primary care practice to call patients within $72 \mathrm{~h}$ of hospital discharge and route problems within the practice for real-time resolution.

PARTICIPANTS: Adult patients with a primary care provider in the general internal medicine practice at the University of California San Francisco who were discharged home from the Medicine service.

MAIN MEASURES: Patients reached directly by phone had a 'full-scripted encounter;' those reached only by voice-mail had a 'message-scripted encounter;' those not reached despite multiple attempts had a 'missed encounter.' Among patients with full-scripted encounters, we identified and cataloged problems arising after hospital discharge and measured the proportion of calls in which a problem was uncovered. For the different encounter types, we compared follow-up appointment attendance and 30-day readmission rates.

KEY RESULTS: Of 790 eligible discharges, 486 had a full-scripted, 229 a message-scripted and 75 a missed encounter. Among the 486 full-scripted encounters, nurses uncovered at least one problem in 371 (76\%) discharges, $25 \%$ of which $(n=94)$ included new symptoms, and $47 \%(n=173)$ included medication issues. Discharges with full-scripted and message-scripted encounters were associated with higher follow-up appointment attendance rates compared with those with missed encounters $(60.1 \%, 58.5 \%, 38.5 \%$ respectively $p=0.004)$. There was no significant difference in 30-day readmission rates $(12.8 \%, 14.8 \%, 14.7 \% ; p=0.72)$.

CONCLUSIONS: Our results suggest that centering a post-discharge phone call program within the primary care practice improves post-hospital care by identifying clinical and care-coordination problems early. With the new Medicare transitional care payment, such pro-

Received November 25, 2013

Revised May 14, 2014

Accepted June 5, 2014

Published online July 24, 2014 grams could become an important, self-sustaining part of the patient-centered medical home.

KEY WORDS: care management; care transitions; health care delivery; primary care; quality improvement.

J Gen Intern Med 29(11):1513-8

DOI: $10.1007 /$ s11606-014-2942-6

(C) Society of General Internal Medicine 2014

\section{INTRODUCTION}

The post-discharge period is a precarious time for discharged patients. ${ }^{1}$ After hospitalization, nearly half of discharged patients will experience at least one error in medication continuity, test follow-up or diagnostic workup, and almost one in five Medicare beneficiaries will be readmitted within 30 days. $^{2,3}$ Interventions to reduce hospital readmissions can be grouped into three domains: pre-discharge interventions (e.g., patient education, medication reconciliation, discharge planning), post-discharge interventions (e.g., follow-up phone calls, timely ambulatory follow-up, post-discharge home visits) and bridging interventions (transition coaches, physician continuity across inpatient and outpatient settings). ${ }^{4}$ In a recent systematic review, no single intervention was found to be associated with a reduced risk for 30-day readmissions. ${ }^{4}$

One intervention that has been tested in multiple settings, including inpatient, primary care and through health insurance plans, is post-discharge follow-up phone calls to the patient. In a Cochrane review focused on follow-up phone calls that originated from the hospital, there was so much diversity in study methodology, outcome measures and patient groups that no conclusion could be drawn on the efficacy of these hospital-based calls. ${ }^{5}$ Previous studies in the primary care setting have focused on readmission outcomes only, and a positive impact on readmissions has not been reliably demonstrated either. ${ }^{6,7}$ Even fewer studies have evaluated the impact of follow-up calls placed through the health plan; however, a recent large study found that patients who received a follow-up phone call through their 
Medicare-managed care plan had a significant reduction in 30-day readmission rates, possibly mediated by an increase in office visits. ${ }^{8}$

Interventions that have been successful at reducing hospital readmissions have frequently bundled interventions. ${ }^{4}$ One successful patient-centered medical home program integrated calls using an interactive voice response (IVR) system post-discharge into a pre-existing intensive case management program. ${ }^{9}$ Despite the limited data on reducing readmissions, the primary care practice is well positioned to address many of the common breakdowns in patient care after hospitalization. For example, while inpatient nurses or health plan-based nurses may review discharge medications, in contrast, the primary care-based nurse can reconcile the discharge medication list with the pre-hospitalization medication list and identify discrepancies. In fact, there is growing support for increased participation and ownership from the primary care practice to coordinate and manage care immediately after hospital discharge. ${ }^{10,11}$ In January 2013, the Centers for Medicare and Medicaid Services (CMS) introduced two new Current Procedural Terminology (CPT) codes, 99495 and 99496, for primary care providers (PCPs) who complete two steps: (1) document discussion with a patient or caregiver about care transitions within 2 days of discharge and (2) have a face-to-face visit with the patient within 2 weeks or 1 week, respectively. ${ }^{10}$ By adding these reimbursable codes, CMS is acknowledging the benefits of timely phone and in-person primary care contact after hospital discharge.

In this study, we describe a quality improvement program whereby nurses embedded in a General Internal Medicine (GIM) practice provide comprehensive review of posthospitalization needs, identify gaps in care and knowledge, and coordinate with practice staff and PCPs to solve any problems identified. Our primary intent was to measure the number and type of problems uncovered by the primary care nurse through phone calls in the immediate postdischarge period. We secondarily evaluated the program's impact on follow-up appointment attendance and 30-day readmission rates. Unlike prior studies that focused on the primary outcome of readmission rates, this article focuses on quantifying and detailing the wide array of problems that arise after hospital discharge and shows how primary care practices can use nursing staff to proactively manage and solve these problems.

\section{METHODS}

\section{Setting}

The intervention was introduced in the GIM practice at the University of California, San Francisco (UCSF), an integrated teaching practice comprised of 31 full- and part-time faculty, 5 fellows and 68 internal medicine residents. The practice manages a population of over 26,500 adult patients, with a mix of private and public insurance. Each month, over 200 GIM patients are discharged from UCSF Medical Center, with an average of 80-120 discharges from the Medicine Service alone. Prior to the intervention, practice staff were tracking discharges from the Medicine Service daily and ensuring GIM patients were offered a follow-up appointment in the practice within 14 days of discharge. The average 30-day readmission rate for the 12 months prior to the intervention was $15.1 \%$.

\section{Intervention}

Between May 2012 and February 2013, two registered nurses (RNs) embedded in the practice called all GIM patients discharged home from the Medicine Service at UCSF Medical Center (main inclusion criteria). In order to focus the program on patients most likely to benefit from a post-discharge nurse phone call, we excluded the following discharges: patient seen or called by PCP prior to first attempted nurse call, patient already readmitted or in the Emergency Department, patient reports no longer seeking primary care at GIM, PCP request to exclude the patient from the nurse call program, patient followed by the physician in the home visit program or patient deceased at time of call. The goal was to call all patients within $72 \mathrm{~h}$ of hospital discharge. During training, the RNs reviewed problem-solving techniques for potential issues that could arise on the call. We developed a workflow to help nurses triage problems to the appropriate practice staff (scheduler, social worker, medical assistant, case manager or PCP). Each morning, the nurses received the call roster from the practice quality analyst. The nurses made up to three attempts to reach the patient or the patient's caregiver. A professional interpreter was used over the telephone when calling non-English speaking patients.

Prior to the start of this intervention, UCSF Medical Center had established two inpatient-based nurse phone call programs: a wrap-around program for heart failure patients and a less detailed call program for Medicine service patients. To prevent duplication and patient confusion, the quality analyst emailed the daily roster of GIM patients each morning to the inpatient nurses who then removed the GIM patients from their scheduled calls, and the GIM patients only received calls from the GIM nurses.

Patients who were reached by phone had a "full-scripted encounter," during which the nurse reviewed all elements of the standardized call script and documented responses in the electronic medical record (EMR) using a structured template and in an Excel spreadsheet that was later used by a research analyst to collate results. In addition to assessing for new or worsening symptoms and confirming homecare needs, nurses engaged in medication reconciliation over the 
phone. If the nurse perceived the medication reconciliation to be too complex over the phone, she scheduled an inperson nursing appointment for medication reconciliation just prior to the patient's follow-up visit. For patients receiving homecare, she contacted the home visiting nurse for assistance with medication reconciliation. The nurses routed all call documentation to the PCP through the EMR. Additional practice staff was copied on the call documentation if they needed to help troubleshoot post-discharge issues. For example, schedulers received messages about needed follow-up appointments. For patients whose PCP is a resident physician, the call documentation was routed to both the resident PCP and that resident's attending physician. The nurses used the Excel spreadsheet to document total time spent preparing for a call (e.g., reviewing the discharge summary in the EMR), on the call and on care coordination after the call.

Patients who could not be reached directly by phone but had voicemail received a "message-scripted encounter," during which the nurse left a message with the call-back number to the practice for questions or concerns and a reminder about any future appointments scheduled in the GIM clinic. A message was left on each attempted call (up to three). If the patient returned the nurse's call and completed the post-discharge encounter, that discharge was subsequently categorized as a "full-scripted encounter." Patients who were never reached despite multiple attempts at all numbers available in the medical record were categorized as a "missed encounter."

\section{Evaluation and Analysis}

Among the full-scripted encounters, the research analyst measured and described the proportion of calls where a problem was uncovered, including new or uncontrolled symptoms, medication issues, homecare, referral, equipment and psycho-social needs, and appointment issues. The analyst reviewed the EMR for documentation of timing when each problem was resolved (e.g., a prescription or referral sent, PCP documentation of resolution) and used these dates to calculate time to problem resolution. In addition, he assessed the proportion of encounters in which the nurses completed medication reconciliation and in which they educated patients as well as the type of education provided.

In addition to describing the results of the full-scripted encounter, we used chi-square bivariate analysis to compare two outcomes according to type of encounter (full-scripted, message-scripted, missed). Our first outcome was postdischarge follow-up appointment attendance rates in the GIM practice. The research analyst reviewed the appointment status in the EMR of all study patients to determine attendance rates. Our second outcome was all-cause 30-day readmission rates. Consistent with CMS methodology, only the first readmission in the 30-day period was counted. We included all inpatient and observation status admissions and excluded patients who died during the index encounter, left against medical advice or transferred to another acute care hospital after the index encounter.

All of our analyses are at the discharge rather than patient level. The study was approved by the UCSF Institutional Review Board.

\section{RESULTS}

Between May 8, 2012 and February 28, 2013, there were 979 discharges home from the Medicine service at UCSF for GIM patients. We excluded 189 discharges because these patients met the exclusion criteria: the majority was already seen in follow-up, had an emergency department visit or been readmitted before a call could be made. Of the 790 remaining eligible discharges (representing 742 patients), 486 had a fullscripted encounter, 229 had a message-scripted encounter and 75 had a missed encounter (Fig. 1).

Among the 486 discharges with full-scripted encounters, nurses uncovered at least one problem in 371 (76\%) discharges. In $25 \%$ of discharges where a problem was uncovered, patients had developed new symptoms since discharge; in $47 \%$ of discharges, the nurses found medication issues, closely split between needing clarification on how to take a medication and needing refills. A true medication error was found in $5 \%$, for example, taking acetaminophen too frequently, putting the patient at risk for liver toxicity, or not taking an antibiotic prescribed to complete pneumonia treatment (Fig. 2). Among discharges where a problem was uncovered, the average number of problems was 2 (range 1-5). Problems were resolved in an average of 2.2 days.

Nurses completed medication reconciliation over the telephone for $272(56 \%)$ discharges. In an additional 58 (12\%), the nurses arranged for in-person medication reconciliation by a GIM nurse, and $46(9 \%)$ more had a homecare nurse complete medication reconciliation, for a $77 \%$ completion rate overall. Among the remaining 114 discharges with no medication reconciliation, 95 did not have their medication list or bottles available at the time of the call, nine refused medication reconciliation, four had a doctor's appointment on the day of the call and two did not have any medications at discharge. In addition to medication reconciliation, the nurses provided patient education for the vast majority (458 discharges, $94 \%$ ) of discharges with full-scripted encounters. Education topics included how to get care urgently (421 discharges, $87 \%$ ), follow-up appointments (373 discharges, $77 \%$ ), medications (294 discharges, $60 \%$ ), symptom management (253 discharges, $52 \%$ ), diet (41 discharges, $8 \%$ ) and home health or social services resources (10 discharges, $2 \%$ ). 


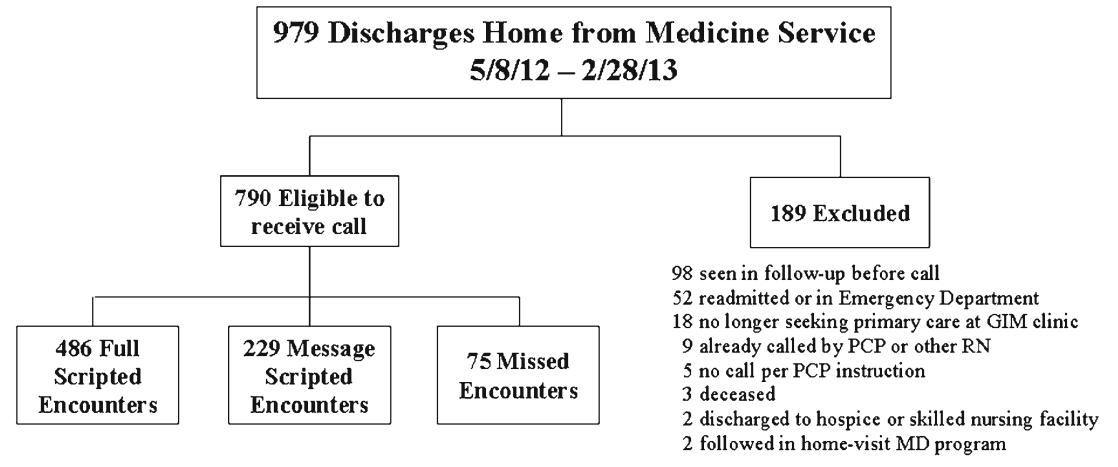

Figure 1. Discharges included in the UCSF General Internal Medicine primary care practice post-discharge nurse phone-call program and type of phone-call received May 2012-February 2013.

Compared with discharges with missed encounters, a significantly higher proportion of those with either a fullscripted encounter or a message-scripted encounter were associated with a completed follow-up appointment in the GIM clinic within 14 days of discharge (respective attendance rates $60.1 \%, 58.5 \%$ and $38.5 \% ; P=0.004$ ).

Discharges with full-scripted encounters trended toward lower readmission rates $(12.8 \%)$ than discharges with either message-scripted (14.8\%) or missed (14.7\%) encounters, although the differences did not reach statistical significance $(P=0.72)$.

The average total times spent per call (preparation, call, post-call care coordination) for full-scripted encounters and message-scripted encounters were 34.1 and $17.6 \mathrm{~min}$, respectively. There was a decreasing trend in total time spent over time.

\section{DISCUSSION}

In this comprehensive primary care-based post-discharge phone call program, nurses reached the majority of patients discharged home and uncovered a substantial number of problems related to symptoms, medications, appointments and unmet service needs. Almost all patients reached had educational needs, ranging from how to obtain urgent care to medication and symptom management. In addition, as problems were uncovered, the nurses served in a carecoordination role, notifying the PCP and clinic staff about the problem and assisting with timely resolution. These results suggest that nurse phone calls based in the primary care practice are effective in improving the quality of care delivered in the immediate post-discharge period.

Patients reached directly or by voicemail had significantly higher attendance rates at follow-up primary care appointments compared to patients whom we could not contact. However, the trend toward a lower 30-day readmission rate was only among those reached directly, suggesting that improved care coordination and real-time problem resolution provided in the first few days after discharge, not just adherence to scheduled follow-up, may be crucial to avoiding unnecessary admissions.

Our program design was most similar to Balaban and colleagues', which tested the impact of a patient discharge form and primary care-based nurse phone calls on several

\section{Uncovered Problems ( $\mathrm{n}=371$ discharges)}

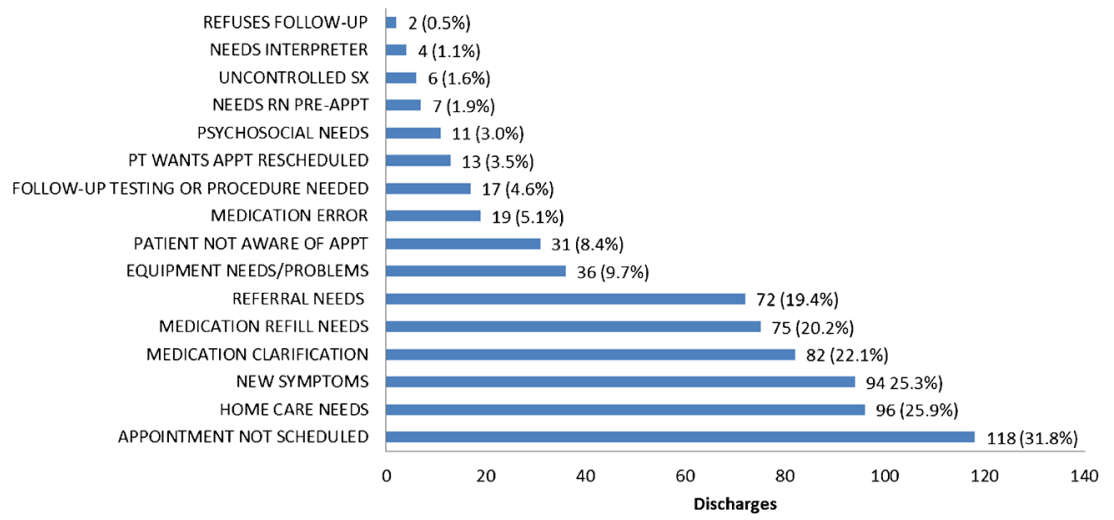

Figure 2. Type of problems uncovered in post-discharge phone call. 
outcome measures. ${ }^{6}$ While their small numbers and relatively low readmission rates precluded detection of a decrease in readmissions, the intervention group completed primary care follow-up and recommended post-hospitalization testing at higher rates. As with our results, their study suggests that the value of basing post-discharge calls in the primary care home serves to improve clinical care in nuanced ways for many more patients than might be observed when only examining readmission rates. Our study complements Balaban's by providing detailed information about the content of the phone calls, thus demonstrating how such a program can improve quality of care for most patients and perhaps avoid unnecessary readmissions for a subset.

The nurse phone call intervention was designed to be comprehensive, covering an extensive list of medical and nonmedical issues that could arise after discharge; because of this comprehensive approach, patient encounters were frequently time consuming. Although our hospital, like many across the country, engages in medication reconciliation at the time of discharge, our nurses noted a substantial amount of time spent on medication reconciliation, particularly for patients with complex regimens and poly-pharmacy. Thus, as the program progressed, we increasingly offered in-person nursing appointments to complete medication reconciliation, usually within a few days of the call, or enlisted the help of the home visiting nurse. This approach led to increased call efficiency; however, it is important to note that quality transitional care may still require an intensive amount of time for medically and socially complex patients who have just been discharged from the hospital. Notably, time spent on message-scripted encounters, while less than full-scripted encounters, was still lengthy because of preparation reading discharge summaries in the EMR and multiple attempts to reach patients. It is possible that these calls could be replaced by an IVR system like that studied by Graham et al. ${ }^{9}$; however, our data suggest that many patients experience substantial problems after hospitalization, and it is not clear how or whether such a program can identify all of the patients with post-discharge needs. Any future studies of an IVR program should pay close attention to whether it is reaching those patients with the most need.

There are several limitations to this study. First, this study was conducted in one academic GIM practice, potentially limiting the applicability of the quality improvement intervention. The practice is located in an urban area and serves an ethnically and culturally diverse population, approximately $15 \%$ of which has limited English proficiency. Our practice also has residents serving as PCPs. Our practice population may be more difficult to access and engage and our structure may make care coordination more difficult than the average primary care practice, suggesting that others may be able to reach their patients after hospitalization with greater ease and efficiency. However, due to their complexity, it is settings and populations such as ours that may most need intensive care-coordination programs to improve care transitions. Additionally, we only included already-established primary care patients who were transitioning out of the hospital back to primary care; patients newly transitioning into primary care but not yet established with a PCP at the time of discharge may have different needs.

This study was not performed as a randomized control trial because it was designed as an evaluation of a quality improvement program for GIM patients discharged home from the Medicine service. While we attempted to reach all patients, there may be patient characteristics that are fundamentally different between those we could and could not contact, which impacted their attendance at follow-up appointments and their risk for readmission. For example, missed encounters may represent patients who are marginally housed or have other psychosocial challenges that make it difficult for our practice nurses to contact them. These same challenges may set them up to miss follow-up appointments in clinic or present back to the hospital with complications after discharge. On the other hand, the nurses informed us that some of the "message-scripted encounters" were due to patients being already fully recovered after hospitalization and back to work. By including these healthier and higher-functioning patients in the "messagescripted encounters" category, the average readmission rate for this group appears lower, and there is a smaller gap between "message-scripted encounters" and "full-scripted encounters."

Finally, we did not find a significant difference in readmission rates among our groups. This may be in part because a proportion of readmissions are not preventable despite excellent care coordination. Although preventing readmissions is important, there are other outcomes, short of readmissions, which are also important to patients and their health, including medication adherence, symptom management and disease control. Our data suggest that these outcomes can be favorably addressed by a primary care-based phone call program in the immediate post-discharge period.

In conclusion, our study supports the value of centering a post-discharge nurse phone-call program in the primary care practice. The success of our program is based on the ability of the nurses to uncover clinical and care-coordination problems in the immediate period after hospitalization and route them appropriately within the practice for rapid resolution. In addition, the reminders and confirmation of follow-up appointments during the calls likely contributed to the higher rate of attendance at those appointments, even for the patients who only received a voice message. Our program is synergistic with the new Medicare transitions of care payment, which requires direct communication with the patient and provision of care coordination within 2 business days of discharge. With these payments supporting proactive care coordination after hospitalization, along with 
a focus on eliciting problems the patient is experiencing, programs such as ours can become an important and selfsustaining part of the patient-centered medical home.

\section{Acknowledgements:}

Contributors: We would like to thank Rosemary Lam for administrative and analytic support, Angela Sherwood and Kelly Flannery for their outstanding nursing and communication skills, and Kathie Buchanan and Eva Turner for their support of the DGIM nurse follow-up phone call program.

Funders: This study was funded by a grant from the Mount Zion Health Fund at UCSF.

Prior Presentations: Presented as a poster at the 2013 Society of General Internal Medicine Annual Meeting on April 26, 2012 and at the 2013 AcademyHealth Annual Research Meeting on June 23, 2013.

Conflict of Interest: The authors declare that they do not have a conflict of interest.

Corresponding Author: Leah Karliner, MD; University of California, San Francisco, 1545 Divisadero Street, Room 232, San Francisco, CA 94115, USA (e-mail: Leah.karliner@ucsf.edu).

\section{REFERENCES}

1. Coleman EA, Berenson RA. Lost in transition: challenges and opportunities for improving the quality of transitional care. Ann Intern Med. 2004;141(7):533-536. PubMed PMID: 15466770.

2. Jencks SF, Williams MV, Coleman EA. Rehospitalizations among patients in the Medicare fee-for-service program. N Engl J Med.
2009;360(14):1418-1428. doi:10.1056/NEJMsa0803563. PubMed PMID: 19339721.

3. Moore C, Wisnivesky J, Williams S, McGinn T. Medical errors related to discontinuity of care from an inpatient to an outpatient setting. J Gen Intern Med. 2003;18(8):646-651. PubMed PMID: 12911647; PubMed Central PMCID: PMC1494907.

4. Hansen LO, Young RS, Hinami K, Leung A, Williams MV. Interventions to reduce 30-day rehospitalization: a systematic review. Ann Intern Med. 2011;155(8):520-528. doi:10.7326/0003-4819-155-8-20111018000008. PubMed PMID: 22007045.

5. Mistiaen P, Poot E. Telephone follow-up, initiated by a hospital-based health professional, for postdischarge problems in patients discharged from hospital to home. Cochrane Database Syst Rev. 2006;4, CD004510. doi:10.1002/14651858.CD004510.pub3. PubMed PMID: 17054207.

6. Balaban RB, Weissman JS, Samuel PA, Woolhandler S. Redefining and redesigning hospital discharge to enhance patient care: a randomized controlled study. J Gen Intern Med. 2008;23(8): 1228-1233. doi:10.1007/s11606-008-0618-9. PubMed PMID: 18452048; PubMed Central PMCID: PMC2517968.

7. Crocker JB, Crocker JT, Greenwald JL. Telephone follow-up as a primary care intervention for postdischarge outcomes improvement: a systematic review. Am J Med. 2012;125(9):915-921. doi:10.1016/ j.amjmed.2012.01.035. PubMed PMID: 22938927.

8. Costantino ME, Frey B, Hall B, Painter P. The Influence of a Postdischarge Intervention on Reducing Hospital Readmissions in a Medicare Population. Popul Health Manag. 2013. doi:10.1089/ pop.2012.0084. PubMed PMID: 23537154

9. Graham J, Tomcavage J, Salek D, Sciandra J, Davis DE, Stewart WF. Postdischarge monitoring using interactive voice response system reduces 30-day readmission rates in a case-managed Medicare population. Med Care. 2012;50(1):50-57. doi:10.1097/MLR.0b013e318229433e. PubMed PMID: 21822152.

10. Bindman AB, Blum JD, Kronick R. Medicare's transitional care payment-a step toward the medical home. N Engl J Med. 2013;368(8):692-694. doi:10.1056/NEJMp1214122. PubMed PMID: 23425161.

11. Tang N. A primary care physician's ideal transitions of care-where's the evidence? J Hosp Med Official Publ Soc Hosp Med. 2013;8(8):472-477. doi:10.1002/jhm.2060. PubMed PMID: 23873732. 